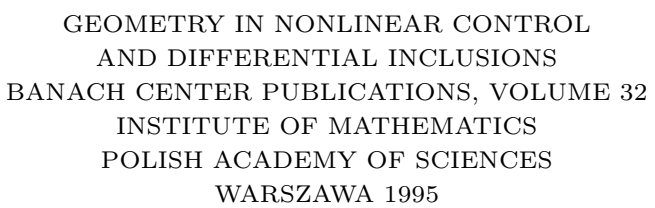

\title{
GLOBAL LINEARIZATION OF NONLINEAR SYSTEMS - A SURVEY
}

\author{
SERGEJ ČELIKOVSKÝ \\ Institute of Information Theory and Automation, Academy of Sciences of the Czech Republic \\ P.O. Box 18, 18208 Prague 8, Czech Republic \\ E-mail: celikovs@utia.cas.cz
}

\begin{abstract}
A survey of the global linearization problem is presented. Known results are divided into two groups: results for general affine nonlinear systems and for bilinear systems. In the latter case stronger results are available. A comparision of various linearizing transformations is performed. Numerous illustrative examples are included.
\end{abstract}

1. Introduction. Exact linearization of nonlinear control systems is probably the most intensively studied and best understood area of the differential geometric approach to the nonlinear control. It presents a natural and challenging task: to find suitable compensations that make a given nonlinear system to behave in a linear fashion, or at least closely to it. This problem deserves a lot of attention: its positive solution directly extends the applicability of the linear methods to a more general nonlinear class of systems. As a consequence, beginning with the pioneering works of Krener [24] and Brockett [3], up to the present time there has been increasing interest in the various modifications of the exact linearization problem. Survey of this field can be found in papers [16,31] or in books [22, 29].

In spite of being aware that all these surveys do not cover many important subfields like time-scaling transformations [33] or dynamic feedback [7, 8], this paper does not aim to give an updated survey of the overall linearization area. It will be concentrated only on the global aspects of the exact linearization since a systematic and self-contained exposition on this topic is still missing.

The number of results on global linearization is rather modest in comparision with the above mentioned linearization boom. The main reason for this is that

1991 Mathematics Subject Classification: $34 \mathrm{H} 05$.

Key words and phrases: exact linearization, global linearization, nonlinear control.

This work was partially supported by the Grant Agency of the Academy of Sciences of the Czech Republic through the Grant 275403.

The paper is in final form and no version of it will be published elsewhere. 
the global linearization problem can not be completely tackled by means of local differential geometry only and requires investigation of the global topological properties of systems in question. In this respect the problem seems to be still open: we will see that presently applied additional topological conditions are relatively simple and straightforward.

Despite the fact that the global linearization is a rather narrow field, we face an important and challenging problem. Progress from the local exact linearization to the global one may be viewed as a natural continuation of the progress from the approximate linearization to the local exact one. At the same time, when applying control scheme "exact linearization plus linear compensator" for a given control goal (e.g. stabilization or tracking) to a particular system, global questions can not be avoided. We will present here several concepts of the globalness and try to discuss their interest from the practical point of view.

In our opinion, existing results on global linearization are mutually rather incongruous. We will therefore try to give an independent and self-contained exposition, supplied when necessary with bibliographical remarks.

Throughout the paper we deal with the affine nonlinear control system of the form

$$
\dot{x}=f(x)+\sum_{k=1}^{m} u_{k} g_{k}(x),
$$

where $x \in M$ (n-dimensional smooth manifold) is the state, $u=\left(u_{1}, \ldots, u_{m}\right)^{\prime} \in$ $R^{m}$ is the input, $f, g_{1}, \ldots, g_{m} \in V(M)$, i.e., they are smooth vector fields $\left({ }^{1}\right)$ on $M$. We do not consider here the output and output relations. Presence of the output relations suggests a further variety of definitions of exact linearization: linearization of the input-output responses only [20,21], linearization of systems with the fixed outputs [15], linearization using output transformations [27], output injection [26], etc. Some global results in this respect may be found in $[27,28,6]$. Such variety would make difficult to point out the typical global aspects that are mainly connected with the properties of the state dynamics of the system.

2. Local and global linearization. Throughout the paper we suppose that the reader is basically familiar with the problem of local exact linearization using various kinds of transformations. Nevertheless, to be self-contained we may sometimes remind some local definitions and results. We do not also consider here all transformations and compensations used at the present time for the linearization and we concentrate ourselves only on three basic and most natural kinds of linearization: state linearization, feedback linearization and restricted feedback linearization.

Definition 1. System (1) is called locally feedback linearizable at $x_{0} \in M$ if

$\left({ }^{1}\right)$ Standard differential geometric notations adopted e.g. in [22] will be used throughout the paper. 
there exist a neighbourhood $U_{x_{0}}$ of $x_{0} \in M$ and a neighbourhood $V_{0}$ of $0 \in R^{n}$, a feedback of the form

$$
u=\alpha(x)+\beta(x) v, \quad \alpha(x) \in C^{\infty}\left(U_{x_{0}}, R^{m}\right), \beta(x) \in C^{\infty}\left(U_{x_{0}}, G l(m, R)\right),
$$

and a diffeomorphism

$$
\mathcal{D}: V_{0} \rightarrow U_{x_{0}}, \quad x=\mathcal{D}(y),
$$

transforming the system (1) into a controllable linear system

$$
\dot{y}=F y+G v, \quad y \in R^{n}, v \in R^{m},
$$

where $F, G$ are $(n \times n)$ and $(n \times m)$ matrices, respectively. The system is called restricted $\left({ }^{2}\right)$ feedback linearizable (state linearizable) if it is feedback linearizable with $\beta(x) \equiv I_{m}\left(\beta(x) \equiv I_{m}, \alpha(x) \equiv 0\right.$, respectively $)$.

Remark 1. Linearization is a particular case of system equivalence: two nonlinear systems are called mutually feedback (restricted feedback, state) equivalent, if they can be transformed into one another using appropriate transformations. Linearizability then means equivalence to a linear controllable system and both terms will be used in the sequel. The same applies to various kinds of global linearization and equivalence that will be introduced later.

Definition 2. System (1) is called globally feedback (restricted feedback, state) linearizable at $x_{0} \in M$ to a linear system on $R^{n}$ if it is locally linearizable at this point and $V_{0}=R^{n}$. It is called globally linearizable on $M$ if $U_{x_{0}}=M$. A system that is linearizable globally on $M$ to a linear system on $R^{n}$ is called globally linearizable.

Remark 2. All types of global linearization introduced by Definition 2 are quite reasonable. Notice that for global state linearization $U_{x_{0}}$ always coincides with the reachable set from $x_{0}$. Of course the case of global linearization (i.e. when both $V_{0}=R^{n}$ and $U_{x_{0}}=M$ ) is most desirable, but at the same time rather restrictive. Linearization at a given $x_{0} \in M$ to a linear system on $R^{n}$ covers cases when a nonlinear system is not globally controllable, but its restriction to the reachable set from $x_{0}$ is globally equivalent to a linear system on $R^{n}$. This case enables a straightforward application of linear methods to solve a particular control goal for the nonlinear system. In the case of global linearization on $M$, one has global controllability of the original nonlinear system, while its linear equivalent is defined on an open subset of $R^{n}$ containing the origin. Nevertheless, this case still remains better than local linearization when $U_{x_{0}}$ is a proper subset of $M$. Finally, let us note that more special and weaker definitions of global linearization may be introduced (see e.g. $[17,14]$ ), nevertheless, their significance seems to be marginal.

We illustrate the above definitions by several examples.

$\left({ }^{2}\right)$ This term is due to [17], Respondek in [32] uses the term "pure feedback". 
EXAMPLE 1. The system

$$
\dot{x}_{1}=x_{1}+u, \quad \dot{x}_{2}=u \exp \left(x_{2}\right),
$$

where $x=\left(x_{1}, x_{2}\right)^{\prime} \in M=R^{2}, u \in R$, is globally state linearizable on $M$ but it is not globally linearizable to a linear system on $R^{2}$. Actually, linearizing at $x_{0}=(0, a)^{\prime}, a \in R$, diffeomorphism is $x=\left(y_{1}+y_{2},-\ln \left(a-y_{1}\right)\right)^{\prime}$ and is defined only for $y_{1}<a$. Nevertheless, its image is the whole $R^{2}$.

EXAMPLE 2. The system

$$
\dot{x}_{1}=x_{1}+u\left(x_{1}+x_{2}\right), \quad \dot{x}_{2}=u x_{2},
$$

where $x=\left(x_{1}, x_{2}\right)^{\prime} \in M=R^{2}, u \in R$, is globally state linearizable to a linear system on $R^{2}$ at any $x_{0} \in\left\{x \in R^{2} \mid x_{2} \neq 0, x_{1}=0\right\}$, but it is not globally linearizable on $M$. Actually, diffeomorphism $\mathcal{D}: R^{2} \rightarrow\left\{x \in R^{2} \mid a x_{2}>0\right\}, \mathcal{D}=$ $\left(a y_{2}+a y_{1}, a\right)^{\prime} \exp y_{1}$, linearizes the system at $x_{0}=(0, a)^{\prime}$. This system is not globally controllable: reachable set from $(0,1)^{\prime}\left(\operatorname{resp} .(0,-1)^{\prime}\right)$ is an open halfplane $x_{2}>0$ (resp. $x_{2}<0$ ).

EXAMPLE 3. The system

$$
\dot{x}=f(x)+u g(x), \quad x \in M=R^{2} \backslash\{0\}, u \in R,
$$

where $f(x)=-(1 / 2) \ln \left(x_{1}^{2}+x_{2}^{2}\right)\left(-x_{2}, x_{1}\right)^{\prime}, g(x)=x$, is not globally linearizable in any sense of Definition 2. The linearizing map is in this case

$$
x=\mathcal{D}(y)=\exp y_{1}\left[\begin{array}{c}
\cos y_{2} \\
\sin y_{2}
\end{array}\right], \quad \mathcal{D}: R^{2} \rightarrow R^{2} \backslash\{0\} .
$$

It is a local diffeomorphism at any $y \in R^{2}$ but is not globally invertible.

EXAMPLE $4([1,13,14])$. The system on $M=R^{2}$

$$
\begin{aligned}
& \dot{x}_{1}=\sin x_{2} \cos x_{2}+u \exp \left(-x_{1}\right) \sin x_{2}, \\
& \dot{x}_{2}=-\left(\sin x_{2}\right)^{2}+u \exp \left(-x_{1}\right) \cos x_{2},
\end{aligned}
$$

is not globally linearizable in any sense of Definition 2. One can easily see that system (3) is transformed into a linear system defined on $R^{2} \backslash\{0\}$ by the smooth map $x=\mathcal{D}(y)$, where

$$
\mathcal{D}^{-1}(x)=\exp x_{1}\left[\begin{array}{c}
\cos x_{2} \\
\sin x_{2}
\end{array}\right], \quad \mathcal{D}^{-1}: R^{2} \rightarrow R^{2} \backslash\{0\},
$$

that is a local diffeomorphism, but not globally one-to-one. Considering the original nonlinear system on $\left\{x=\left(x_{1}, x_{2}\right)^{\prime} \in R^{2} \mid x_{2} \in(-\pi / 2, \pi / 2)\right\}$ and the linearized one on $R^{2} \backslash\left\{y=\left(y_{1}, y_{2}\right)^{\prime} \in R^{2} \mid y_{1}>0\right\}$ we obtain one-to-one correspondence. Note that both these sets are not invariant with respect to the corresponding systems.

ExAmple 5. The system on $M=R^{2}$

$$
\dot{x}_{1}=2 x_{1}+u\left(1+x_{2}\right), \quad \dot{x}_{2}=x_{2}+u,
$$


is globally state linearizable since its linearizing diffeomorphism $x=\mathcal{D}(y)=$ $\left(y_{1}+(1 / 2) y_{2}^{2}, y_{2}\right)^{\prime}$ is the global diffeomorphism of $R^{2}$ onto itself.

In the previous examples we concentrated only on the state linearization case in order to illustrate briefly the main obstructions for the global linearization of systems that are locally everywhere linearizable. In Example 1 one can observe that the vector field $\left(1, \exp x_{2}\right)^{\prime}$ is not complete, i.e. its integral curves are not defined for all time moments. We will see in the sequel that the completeness of a certain collection of vector fields is necessary for the linearized system to be defined on the whole $R^{n}$. Example 3 illustrates another basic topological property necessary for the global linearization: the simple connectedness of $M$ that is obviously violated here. Simple connectedness usually guarantees that the linearizing diffeomorphism is globally one-to-one. Nevertheless, as indicated by Example 4, it is not true that the system, locally everywhere linearizable on a simply connected manifold, is globally linearizable on $M$.

3. General results. In this section available results about general nonlinear systems of the form (1) will be presented. Most of them deal either with state linearization or restricted feedback linearization. The reason is that, contrary to the case of the unrestricted feedback, linearizing restricted feedback (when existing) is unique (up to the form of the resulting linear system). As a consequence, the results on restricted feedback linearization can be also used to exclude global linearizability of a particular nonlinear system.

Another typical feature of what we call here general results is that they are not easy to be applied to a particular system. The reason is that many of them may be viewed as rather straightforward reformulation of the definition of global linearization. For instance, often one can meet requirement that a certain map should be globally one-to-one ([17], Theorem 3 or [14], Theorems 1-3). Actually, proving the global bijectivity of a locally diffeomorphic map is the key issue of the global linearization and also an open general mathematical problem.

3.1. State and restricted feedback linearization. Global state linearization is solved by the following theorem.

THEOREM 1. System (1) is globally state linearizable if and only if there exist (after a reordering the inputs) Kronecker indices $\mu_{1} \geq \ldots \geq \mu_{m} \geq 0, \sum_{k=1}^{m} \mu_{k}=$ $n$, such that

1) $\forall x \in M \operatorname{dim} \operatorname{span}\left\{\operatorname{ad}_{f}^{i} g_{k}(x) \mid 1 \leq k \leq m, 0 \leq i \leq \mu_{k}-1\right\}=n$,

2) $\forall x \in M\left[\operatorname{ad}_{f}^{p} g_{k}(x), \operatorname{ad}_{f}^{q} g_{l}(x)\right]=0,1 \leq k, l \leq m, 0 \leq p+q \leq \mu_{k}+\mu_{l}-1$,

3) $\exists x_{0} \in M: f\left(x_{0}\right)=0$,

4) the vector fields $f, g_{k}, k=1, \ldots, m$, are complete,

5) $M$ is simply connected.

Corollary 1. System (1) is globally state linearizable at $x_{0} \in M$ to a linear system on $R^{n}$ if and only if there exist (after reordering the inputs) Kronecker 
indices $\mu_{1} \geq \ldots \geq \mu_{m} \geq 0, \sum_{k=1}^{m} \mu_{k}=n$, such that

1) there exists a largest connected set $N \ni x_{0}$ such that $\forall x \in N$

$$
\operatorname{dim} \operatorname{span}\left\{\operatorname{ad}_{f}^{i} g_{k}(x) \mid 1 \leq k \leq m, 0 \leq i \leq \mu_{k}-1\right\}=n
$$

2) $\forall x \in N\left[\operatorname{ad}_{f}^{p} g_{k}(x), \operatorname{ad}_{f}^{q} g_{l}(x)\right]=0,1 \leq k, l \leq m, 0 \leq p+q \leq \mu_{k}+\mu_{l}-1$,

3) $f\left(x_{0}\right)=0$,

4) the vector fields $f, g_{k}, k=1, \ldots, m$ are complete on $N$,

5) $N$ is simply connected.

Remark 3. Conditions 1-3 of Theorem 1 being valid in a neighbourhood of $x_{0}$ are necessary and sufficient for the local state linearization ([24]). Condition 4 is e.g. valid when $\left\|f, g_{k}(x)\right\| \leq C(1+\|x\|)$ for a suitable constant $C \geq 0$. Condition 5 of Theorem 1 can be verified easily, but the situation considered there is rather rare. More typical is the situation of Corollary 1 and when $N$ is a proper subset of $M$, then the Condition 5 is in general very difficult to check. Moreover, conditions 1-4 imply the existence of the smooth regular map $\mathcal{D}: R^{n} \rightarrow M$ such that $N=\mathcal{D}\left(R^{n}\right)$ and $\mathcal{D}$ locally linearizes the nonlinear system at any $x \in N$. For this map there is a very straightforward equivalence between $\mathcal{D}$ being one-to-one and $N$ being simply connected. In other words, checking the simple conectedness of $N$ is as difficult as the direct establishing of the global invertibility of a linearizing local diffeomorphism.

Remark 4. The proof of Theorem 1 (Corollary 1 is its obvious consequence) may be performed in several ways. Assuming analyticity of system data Respondek [32] uses a certain global theorem introduced to control theory by Sussmann [35] and Krener [24, 25]. For the $C^{\infty}$ data one can easily adapt the proof used by Dayawansa et al. in [17] (actually, it is applicable even in $C^{n+1}$ case). It is based on the fact that the map $\mathcal{D}: R^{n} \rightarrow M$ defined as

$$
\mathcal{D}(y)=\Phi_{n}^{y_{n}} \circ \ldots \circ \Phi_{1}^{y_{1}}\left(x_{0}\right),
$$

where $\Phi_{i}^{t}, i=1, \ldots, n$, are mutually commuting flows of vector fields $\operatorname{ad}_{f}^{j} g_{k}$, $k=1, \ldots, m, 0 \leq j \leq \mu_{k}-1$, is by conditions 1,4 a local diffeomorphism at any $y=\left(y_{1}, \ldots, y_{n}\right)^{\prime} \in R^{n}$ and by conditions 2,3 linearizes system (1). At the same time it defines a transitive abelian Lie group action on $M$ and therefore $\mathcal{D}$ is globally one-to-one if and only if $M$ is simply connected. Actually, it is appropriate to note here that the last fact is a consequence of the local invertibility of $\mathcal{D}$ at $\forall y \in$ $R^{n}$ and $\mathcal{D}\left(R^{n}\right)=M$ only (any covering of a simply connected set is one-to-one). The fact that $\mathcal{D}$ defines transitive abelian Lie group action implies in particular that $\mathcal{D}\left(R^{n}\right)=M$, but it is not necessary for the last equality. Another useful observation is that Respondek uses completeness of $f, g_{i}$ in order to prove (based on Palais [30]) completeness of vector fields $\operatorname{ad}_{f}^{j} g_{k}, k=1, \ldots, m, 0 \leq j \leq \mu_{k}-1$ while Dayawansa et al. [17] consider the last fact as an assumption. As it will be seen in Example 6, paradoxically it may be in some cases more simple to check this assumption. 
R e mark 5. One can easily see using the above scheme that if all conditions of Theorem 1 hold with the exception of the existence of a point $x_{0} \in M$ with $f\left(x_{0}\right)=0$, then the corresponding system is globally state equivalent to a system

$$
\dot{y}=F y+G u+d, \quad d \in R^{n},
$$

$(F, G)$ being a controllable pair. Linear controllability implies that there exist a shift of the $y$-space and a constant (in $y$-coordinates) feedback removing $d$ from the above equation. This indicates that in the case of any kind of global feedback linearization one need not worry about the existence of an equilibrium point. Note that this is possible only in the global case since in the local case an arbitrary shift is not available. A similar observation applies to global linearization to a linear system on $R^{n}$.

For the restricted feedback linearization, we concentrate ourselves on the single-input case, since multi-input results are not available. The reason is probably that even the conditions for the local restricted feedback linearization are in the multi-input case rather complicated (see [31], Theorem 3.3).

Global restricted feedback linearization results are rather straightforward generalizations of the state linearization case. The reason is that there exists (if any) a unique restricted feedback $\alpha(x)$ such that the corresponding closed loop system is state equivalent to a linear system in Brunovský canonical form (5]). Moreover, this feedback can be computed using a straighforward, linear algebraic scheme. As the result, extra global requirements are very similar to those of Theorem 1.

To be more precise, let us consider a single input nonlinear system of the form (1), i.e. with $m=1$ and $g_{1}=g$. Let us define the following distributions:

$$
D^{i}(x)=\operatorname{span}\left\{\operatorname{ad}_{f}^{j} g(x), j=0, \ldots, i\right\}, \quad i=0, \ldots, n-1 .
$$

At any $x \in M$ such that $\operatorname{dim} D^{n-1}(x)=n$ we may define (and easily compute by purely linear algebraic methods) the unique one-form $\eta$ as

$$
\eta\left(\operatorname{ad}_{f}^{i} g\right)=(-1)^{n-1} \delta_{i, n-1}, \quad i=0,1, \ldots, n-1 .
$$

Finally, let

$$
\alpha(x)=-L_{f}^{n-1}(\eta(f))(x),
$$

then it is known from the local theory that for any restricted feedback linearizable single-input system $\alpha$, with the above mentioned properties, is unique. For the global case we have

Theorem 2. A single input system (1) is globally restricted feedback linearizable if and only if $\forall x \in M$ (see $(7,6)$ for $\alpha$ and $(5)$ for $\left.D^{i}\right)$

1) $\operatorname{dim} D^{n-1}(x)=n$,

2) (a) $\eta$ is an exact one-form or equivalently

(b) $\left[D^{n-1}, D^{n-1}\right] \subset D^{n-2}$ or equivalently

(c) $\left[g, \mathrm{ad}_{f+\alpha g}^{j} g\right]=0 \forall j=1,3, \ldots 2 n-1$ 
3) the vector fields $g, f+\alpha g$ are complete,

4) $M$ is simply connected.

COROllary 2. Let us consider arbitrary $x_{0} \in R^{n}$ and let $N \subset M, x_{0} \in N$ be the largest connected open set with respect to inclusion such that condition 1 of Theorem 2 holds $\forall x \in N$. A single-input system (1) is globally restricted feedback linearizable at $x_{0} \in R^{n}$ to a linear system on $R^{n}$ if and only if $N$ is simply connected and conditions 2, 3 hold on $N$.

The proof of Theorem 2 (Corollary 2 is its easy consequence) can be performed slightly adapting proofs of the similar results in [32] (for analytic case) or in [17] for $C^{\infty}$ (more exactly, even for $C^{2 n+2}$ ) case. Actually, our Theorem 2 is a mixture of formulations used in the mentioned references. It is appropriate to note that the arguments generalizing Theorem 1 to Theorem 2 are identical with the arguments used for the well know local result on restricted feedback linearization: the the above feedback $v=\alpha+u$ is well defined at any point and transforms the corresponding system to the case when Theorem 1 is applicable.

Example 6. Consider bilinear system

$$
\dot{x}_{1}=u x_{2} \quad \dot{x}_{2}=x_{1}
$$

where $x=\left(x_{1}, x_{2}\right)^{\prime} \in R^{2}$. We have $f=\left(0, x_{1}\right)^{\prime}, g=\left(x_{2}, 0\right)^{\prime},[f, g]=\left(x_{1},-x_{2}\right)^{\prime}$ so that $\operatorname{dim} D^{1}(x)=2$ for $\forall x_{2} \neq 0$. Consider $N=\left\{x \in R^{2} \mid x_{2}>0\right\}$ (the case $N=\left\{x \in R^{2} \mid x_{2}<0\right\}$ is analogical). We have obviously on $N$ the exact form $\eta=\left(0,1 / x_{2}\right)^{\prime}, \eta(g)=0, \eta([f, g])=-1$, feedback $\alpha=x_{1}^{2} / x_{2}^{2}$ and $f+\alpha g=$ $\left(x_{1}^{2} / x_{2}, x_{1}\right)^{\prime}$ is a complete vector field on the simply connected set $N$. By Corollary 2 the above system is globally restricted feedback linearizable at any $x_{0} \in N$ to a controllable linear system on $R^{n}$. Straightforward computations show that the linearizing diffeomorphism is $x=\exp \left(y_{1}\right)\left(y_{2}, 1\right)^{\prime}$. Notice also that the original bilinear system was globally controllable, i.e. $N$ considered in Corollary 2 need not be equal to the reachable set from $x_{0} \in N$.

The above example illustrates that checking the completeness may be in general very difficult task, since the sufficient condidion mentioned in Remark 3 is not valid here and completeness of the vector field $f+\alpha g$ should be checked in a different way (we left it to the interested reader). Notice that $[f+\alpha g, g]=-x$ is linear and thus complete. This shows that in some cases (especially for low dimensions) it may be reasonable to check completeness of $g,[f+\alpha g, g], \ldots$ rather than that of $f+\alpha g$ itself.

3.2. Unrestricted feedback. As it was already indicated, the case of the unrestricted feedback is complicated due to the fact that the linearization need not be unique. First, we give two examples to illustrate this difficulty.

EXAMPLE 7. Consider the following planar system with two inputs:

$$
\dot{x}=\exp \left(-x_{1}\right)\left(\left(\cos x_{2},-\sin x_{2}\right)^{\prime} u_{1}+\left(\sin x_{2}, \cos x_{2}\right)^{\prime} u_{2}\right) \text {. }
$$


Both mutually commuting vector fields on the right-hand side of this system are not complete, the system is locally state linearizable around any point, but it is not globally state linearizable in any sense of Definition 2. On the other hand, the above system is easily globally linearizable using the unrestricted feedback:

$$
v=\left[\begin{array}{l}
v_{1} \\
v_{2}
\end{array}\right]=\exp \left(-x_{1}\right)\left[\begin{array}{cc}
\cos x_{2} & \sin x_{2} \\
-\sin x_{2} & \cos x_{2}
\end{array}\right]\left[\begin{array}{l}
u_{1} \\
u_{2}
\end{array}\right]
$$

The next example is formulated as a Proposition since it describes a fairly general planar situation.

Proposition 1. Consider the planar single-input system of the form

$$
\begin{aligned}
& \dot{x}_{1}=u, \\
& \dot{x}_{2}=f_{0}\left(x_{2}\right)+f_{1}\left(x_{2}\right) x_{1}, \quad f_{0}(0)=0 .
\end{aligned}
$$

It is

1) locally both restricted and unrestricted feedback linearizable around the origin if and only if $f_{1}(0) \neq 0$;

2) globally restricted feedback linearizable if and only if $f_{1}\left(x_{2}\right) \neq 0 \forall x_{2} \in R$ and $\left(0, f_{1}\right)^{\prime}$ is a complete vector field;

3) globally unrestricted feedback linearizable if and only if $f_{1}\left(x_{2}\right) \neq 0 \quad \forall x_{2} \in R$.

Pr o of. Parts 1, 2 follow from Theorem 2 and the corresponding local results (see $[23,3])$. To prove the remaining one, let us observe that $y=\left(f_{1}\left(x_{2}\right) x_{1}+\right.$ $\left.f_{0}\left(x_{2}\right), x_{2}\right)^{\prime}$ is a global diffeomorphism of $R^{n}$ that together with the feedback $v=f_{1} u+\left(f_{0}^{\prime}+f_{1}^{\prime} x_{1}\right)\left(f_{1} x_{1}+f_{0}\right)$ linearizes the above system.

Observe that for the class of systems considered in Proposition 1 locally there is no difference between restricted and unrestricted feedback linearizability (of course in each case the system is linearized using different transformations). At the same time, application of the unrestricted feedback substantially enlarges possibilities for the global linearization.

In [19] sufficient conditions for the unrestricted feedback linearization of a single-input system are proved. They apply several known results about global invertibility of mappings from $R^{n}$ to $R^{n}$. The paper of Cheng et al. [14] treats multiinput case and gives necessary and sufficient conditions, that unfortunatelly contain a priori requirement of the global invertibility of a certain map. Boothby [2] gives necessary and sufficient conditions in terms of the global integrability of the system on a simply connected manifold and the completeness of vector fields modified by the linearizing feedback. Special attention is devoted to the twodimensional cases. Similar results are obtained in Dayawansa et al. [18].

We give here a rather different formulation of the necessary and sufficient conditions for the restricted feedback linearization that is, nevertheless, equivalent to the single-input version of [14] and we indicate its relation to the results of [2, 18]. For the simplicity we restrict ourselves to the single-input case. 
THEOREM 3. Single-input nonlinear system (1) is globally unrestricted feedback linearizable on $M$ if and only if it is globally state equivalent to the following system

$$
\begin{gathered}
\dot{x}=f(x)+g(x) u, \quad x=\left(x_{1}, \ldots, x_{n}\right)^{\prime} \in R^{n} \\
g=\left(g_{1}(x), 0, \ldots, 0\right)^{\prime}, f=\left(f_{1}(x), f_{2}(x), f_{3}\left(x_{2}, \ldots, x_{n}\right), \ldots, f_{n}\left(x_{n-1}, x_{n}\right)\right)^{\prime}
\end{gathered}
$$

and

$$
g_{1}(x) \neq 0, \quad \frac{\partial f_{j}}{\partial x_{j-1}} \neq 0 \quad \forall x \in R^{n}, j=2, \ldots, n .
$$

If in addition there exists a constant $\varepsilon>0$ such that

$$
\left|\frac{\partial f_{j}}{\partial x_{j-1}}\right|>\varepsilon \quad \forall x \in R^{n}, j=2, \ldots, n,
$$

then the above system is globaly unrestricted feedback linearizable, i.e. the corresponding linear system is defined on the whole $R^{n}$.

Remark 6. Nonlinear single-input system is globally state equivalent to the form (8) if and only if distributions $D^{i}, i=0, \ldots, n-1$, (see (5) for their definition) can be transformed using a global coordinate change into the form $D^{i}=\operatorname{span}\left\{\partial / \partial x_{j}, j=0, \ldots, i\right\}, i=0, \ldots, n-1$ (we will call this property as the global integrability of a nested sequence of distributions). Necessary conditions for this are the involutivity of $D^{i}$ and $\operatorname{dim} D^{i}(x)=i+1, \forall i=0,1, \ldots, n-1$, $x \in M$ (in the local case they are also sufficient - see [23]), but they are not sufficient. Cheng et al. [14] therefore requires existence of linearly independent vector fields $Z_{i} \in D^{i}, i=0, \ldots, n-1$, such that

$$
y=\left(y_{1}, \ldots, y_{n}\right) \rightarrow \Phi_{Z_{1}}^{y_{1}} \circ \ldots \circ \Phi_{Z_{n-1}}^{y_{n}}\left(x_{0}\right)
$$

is a bijection of $R^{n}$ onto $M$ (actually, this bijection defines a global coordinate change taking the investigated nonlinear system to the special form (8)). One can really expect the last assumption to be difficult to check, moreover, as indicated by the previous examples, the above map may be globally bijective for some selections of $Z_{i}$ 's while for other selections it need not be. For the multi-input case, treated analogically, the reader is referred to [14] as well as for the full proof.

R e mark 7. Proof of Theorem 3 is the constructive one, i.e. it gives an algorithm for explicit computations of all linearizing transformations. It computes at first the linearizing diffeomorphism and then the appropriate unrestricted feedback. Let us also note that the condition

$$
\left|\frac{\partial f_{j}}{\partial x_{j-1}}\right|>\varepsilon \quad \forall x \in R^{n}, j=2, \ldots, n,
$$

is only sufficient for the linearized system to be defined on the whole $R^{n}$ (it is not necessary - see e.g. globally linearizable system $\left.\dot{x}_{1}=u, \dot{x}_{2}=\exp \left(-x_{2}\right) x_{1}\right)$. When comparing Theorem 3 with the results of $[2,18]$ we can observe that the above 
sufficient condition is also the sufficient condition for the completeness of the appropriate collection of vector fields. Results of $[2,18]$ are therefore more general than Theorem 3; nevertheless, since no criterion for completeness is known, the difference is not so important.

4. Bilinear systems. Here we present results for a more particular class of nonlinear systems - bilinear systems. It turns out that for this class it is possible in some cases to remove general a priori type assumptions and even obtain the equivalence between local and global linearization.

Consider bilinear system of the form

$$
\dot{x}=\left(A+\sum_{k=1}^{m} B_{k} u_{k}\right) x+C u .
$$

Here $x=\left(x_{1}, \ldots, x_{n}\right)^{\prime} \in R^{n}$ is the state, $u=\left(u_{1}, \ldots, u_{m}\right)^{\prime} \in R^{m}$ is the input; $A, B_{k}, C$ are real matrices of appropriate dimensions.

We start with global state linearization. A single-input (homogeneous) bilinear system has the form (9) with $k=1, B_{1}=B, C=c \in R^{n}(C=0)$. The following two theorems are given in [10].

THEOREM 4. A single-input bilinear system (9) is globally state linearizable at $x_{0} \in R^{n}$ to a linear system on $R^{n}$ if and only if it is locally state linearizable at $x_{0}$.

THEOREM 5. Let $\mathcal{A}^{k} \subset R^{n}, k \leq n$, be the $k$-dimensional affine submanifold given by $E x=d, d \in R^{n}$, with $E$ being an $(n-k) \times n$ matrix of full rank. Providing that $\mathcal{A}^{k}$ is invariant with respect to (9) consider restriction of (9) to $\mathcal{A}^{k}$. Then this restriction is globally state linearizable at $x_{0} \in \mathcal{A}^{k}$ to a linear system on $R^{k}$ if and only if it is locally linearizable at $x_{0}$.

Remark 8. Straightforward evaluation shows that Theorems 4,5 are mutually equivalent. This is based on the rather old trick going back to Brockett [4], when nonhomogeneous bilinear system can be considered as a homogeneous one in the state space of higher dimension (column vectors of $C$ are additional states). The aim of Theorem 5 is two-fold. First, it can be proved more easily than Theorem 4 based on properties of a matrix (in particular, finite dimensional) Lie algebra (see $[9,10]$ ). Secondly, it gives an answer about global linearizability for systems defined on $R^{n}$, but actually evolving on a lower dimensional submanifold.

Examples of $n$-dimensional single-input homogeneous bilinear systems (9) that are globally linearizable at an equilibrium point are given in [9]. The same reference presents a complete list of all single-input homogeneous bilinear systems on $R^{2}$ and $R^{3}$ that are globally linearizable at an equilibrium point (a typical planar case is our Example 2). Finally, one can find in [10] examples of $n$-dimensional nonhomogeneous bilinear systems that are globally state linearizable (i.e. by a global diffeomorphism of $R^{n}$ onto itself)—see also our Example 5. 
ExAmple 8. We show that Theorem 4 cannot be generalized to the multi-input case. Consider the system

$$
\dot{x}=\left(u_{1}\left[\begin{array}{ll}
1 & 0 \\
0 & 1
\end{array}\right]+u_{2}\left[\begin{array}{cc}
0 & 1 \\
-1 & 0
\end{array}\right]\right) x
$$

that is obviously locally state linearizable at any point except the origin but it is not globally feedback linearizable in any sense of Definition 2 since it evolves on $R^{2} \backslash\{0\}$.

An additional sufficient condition for the global state linearization of multiinput nonhomogeneous bilinear systems were derived in the recent work [12]. This condition consists in the requirement that all nonzero linear combinations of $B_{1}, \ldots, B_{m}$ do have an eigenvalue distinct from $2 \pi k \sqrt{-1}, k$ integer. Complete analysis of state linearizable multi-input bilinear systems with state space dimensions up to 5 is also provided there.

An interesting question arises: is it possible to extend the previous equivalence between the local and global linearizations of bilinear systems also to the case of the (restricted) feedback linearization? Actually, we loose in this case most of the advantages of bilinear systems, namely, guaranteed completeness of all vector fields and application of properties of matrix Lie algebras. As a consequence, only results in small dimension are available for restricted feedback while for the unrestricted feedback a counterexample indicates the negative answer to the above question.

THEOREM 6. Consider a homogeneous single-input bilinear system in $R^{2}$ or $R^{3}$. It is globally restricted feedback linearizable to a linear system on $R^{2}$ or $R^{3}$, respectively, if and only if it is locally restricted feedback linearizable.

Remark 9. Previous theorem is given in [11]. Actually, a complete list of both the locally and globally restricted feedback linearizable homogeneous singleinput bilinear systems is given there. A typical two-dimensional case is indicated by the earlier Example 6.

EXAMPLE 9. We show that for the unrestricted feedback case there exists a locally linearizable single-input bilinear system that is not globally linearizable in any sense. Consider the following system

$$
\dot{x}=\left(\left[\begin{array}{ll}
2 & 2 \\
1 & 1
\end{array}\right]+u\left[\begin{array}{ll}
2 & 0 \\
0 & 1
\end{array}\right]\right) x
$$

with $x=\left(x_{1}, x_{2}\right)^{\prime} \in R^{2}, u \in R$, around its equilibrium $(1,-1)^{\prime}$. Obviously

$$
[A, B]=\left[\begin{array}{cc}
0 & -2 \\
1 & 0
\end{array}\right],
$$

i.e. $\quad B x,[A, B] x$ are linearly independent $\forall x \in R^{2} \backslash\{0\}$ and the system is locally unrestricted feedback linearizable around the mentioned equilibrium. At the same time it is not globally linearizable in any sense of Definition 2. Actually, it should 
hold for the possible linearizing diffeomorphism $\mathcal{D}$ that $\mathcal{D}\left(R^{2}\right)=R^{2} \backslash\{0\}$ and therefore either $\mathcal{D}$ is not one-to-one or resulting linear system is not defined on the whole $R^{n}$.

5. Conclusions. An attempt was made to present briefly a collection of known results in the field of the global linearization of nonlinear systems. Two groups of results are described. First, results on general affine nonlinear systems are given which use additional general topological assumptions to tackle the problem. Secondly, results are provided which try to find more particular classes of systems for which general topological assumptions may be avoided. In this context a question arises, namely, what are the perspectives of the further development in this field?

As indicated at the beginning of the paper, only two simple conditions were used in addition to the local linearizability: simple connectedness and completeness. On the general level it seems to be therefore reasonable to try to engage more topology to study the problem. For instance, the following question seems to be challenging: does there exist a smooth (analytic) single-input system with $M=R^{n}$ satisfying all conditions of Corollary 1 except the last one? At least, no example of such system is known to the author (recall Example 3-the vector field $f(x)$ is not smooth at $\left.0 \in R^{2}\right)$. Notice that for multi-input case such a system exists - see Example 8. A negative answer to this question would lead to the generalization of Theorem 4 to the class of nonlinear systems defined on $R^{n}$ by smooth (analytic) complete vector fields.

Also the second group of results suggests open problems, e.g. restricted feedback linearization of $n$-dimensional bilinear systems or further analysis of the multi-input case.

Acknowledgments. The author wishes to express his gratitude to the editors of this volume as well as to the anonymous referees for their helpful suggestions which have greatly improved the final version of this paper.

\section{References}

[1] W. M. Boothby, Some comments on global linearization of nonlinear systems, Systems Control Lett. 4 (1984), 143-149.

[2] —, Global feedback linearizability of locally linearizable systems, in: Algebraic and Geometric Methods in Nonlinear Control Theory, M. Fliess and M. Hazewinkel (eds.), Reidel, Dordrecht, 1986, 243-246.

[3] R. W. Brockett, Feedback invariants for nonlinear systems, Prepr. IFAC Congr., Helsinki, Finland, 1978, 1115-1120.

[4] -, On the algebraic structure of bilinear systems, in: Theory and Applications of Variable Structure Systems, R. R. Mohler and A. Ruberti (eds.), Academic Press, New York, 1972, 153-168.

[5] P. Brunovský, A classification of linear controllable systems, Kybernetika 1970, 173180. 
[6] C. Byrnes and A. Isidori, Asymptotic stabilization of minimal phase nonlinear systems, IEEE Trans. Automat. Contr. 36 (1991), 1122-1137.

[7] B. Charlet, J. Lévine and R. Marino, On dynamic feedback linearization, Systems Control Lett. 13 (1989), 143-151.

[8] —, - , - Sufficient conditions for dynamic state feedback linearization, SIAM J. Control Optim. 29 (1991), 38-57.

[9] S. Čelikovský, On the global linearization of bilinear systems, Systems Control Lett. 15 (1990), 433-439.

[10] - On the global linearization of nonhomogeneous bilinear systems, ibid. 18 (1992), 397-402.

[11] - On the relation between local and global linearization of bilinear systems, in: Systems Structure and Control, V. Strejc (ed.), Pergamon Press, Oxford, 1992, 172-175.

[12] —, Global state linearization of multi-input bilinear systems, in: Proc. 1st Asian Control Conf., Tokyo, July 1994, Vol. 3, 133-136.

[13] D. Cheng, T. J. Tarn and A. Isidori, Global linearization of nonlinear systems, in: Proc. 23rd. IEEE Conference on Decision and Control, 1984, 74-83.

[14] - - - - Global external linearization of nonlinear systems via feedback, IEEE Trans. Automat Control AC-30 (1985), 808-811.

[15] D. Cheng, A. Isidori, W. Respondek and T. J. Tarn, Exact linearization of nonlinear systems with outputs, Math. Systems Theory, 21 (1988), 63-83.

[16] D. Claude, Everything you always wanted to know about linearization but were afraid to ask, in: Algebraic and Geometric Methods in Nonlinear Control Theory, M. Fliess and M. Hazewinkel (eds.), Reidel, Dordrecht, 1986, 181-226.

[17] W. Dayawansa, W. M. Boothby and D. L. Elliot, Global state and feedback equivalence of nonlinear systems, Systems Control Lett. 6 (1985), 229-234.

[18] _ - - - - Global linearization by feedback and state transformations, in: Proc. 24th IEEE Conf. on Decision and Control, Dec. 1985, 1042-1049.

[19] L. R. Hunt, R. Su and G. Meyer, Global transformation of nonlinear systems, IEEE Trans. Automat. Control AC-28 (1983), 24-31.

[20] A. Isidori, J. A. Krener, C. Gori Giorgi and S. Monaco, Nonlinear Decoupling via feedback: a differential geometric approach, IEEE Trans. Automat. Control AC-26 (1981), 331-345.

[21] A. Isidori and A. Ruberti, On the synthesis of linear input-output responses for nonlinear systems, Systems Control Lett. 4 (1984), 17-22.

[22] A. Isidori, Nonlinear Control Systems: An Introduction, 2nd ed., Springer, Berlin, 1989.

[23] B. Jakubczyk and W. Respondek, On linearization of control systems, Bull. Acad. Polon. Sci. Sér. Sci. Math. 28 (1980), 517-522.

[24] A. J. Krener, On the equivalence of control systems and the linearization of nonlinear systems, SIAM J. Control Optim. 11 (1973), 670-676.

[25] A. J. Krener, A decomposition theory for differentiable systems, ibid. 15 (1977), 813829.

[26] A. J. Krener and A. Isidori, Linearization by output injection and nonlinear oservers, Systems Control Lett. 3 (1983), 47-52.

[27] R. Marino, W. Respondek and A. J. van der Shaft, Equivalence of nonlinear control systems to input-output prime forms, SIAM J. Control Optim. 32 (1994), 387407.

[28] - , - - - Almost disturbance decoupling for single-input single output nonlinear systems, IEEE Trans. Automat. Control 34 (1989), 1013-1017.

[29] H. Nijmeijer and A. J. van der Shaft, Nonlinear Dynamical Control Systems, Springer, Berlin, 1990. 
[30] R. S. Palais, A global formulation of the Lie theory of transformation groups, Mem. Amer. Math. Soc. 22 (1957).

[31] W. Respondek, Geometric methods in linearization of control systems, in: Mathematical Control Theory, Banach Center Publ. 14, PWN Warszawa, 1985, 453-467.

[32] - Global aspects of linearization, equivalence to polynomial forms and decomposition of nonlinear systems, in: Algebraic and Geometric Methods in Nonlinear Control Theory, M. Fliess and M. Hazewinkel (eds.), Reidel, Dordrecht, 1986, 257-284.

[33] M. Sampei and K. Furuta, On time scaling for nonlinear systems: application to linearization, IEEE Trans. Automat. Control AC-31 (1986), 459-462.

[34] R. Su (1982), On the linear equivalents of nonlinear systems, Systems Control Lett. 2 (1982), 48-52.

[35] H. J. Sussmann, An extension of a theorem of Nagano on transitive Lie algebras, Proc. Amer. Math. Soc. 45 (1974), 349-356. 